\title{
Distorsión de la imagen corporal y trastornos alimentarios en adolescentes gimnastas respecto a un grupo control de adolescentes no gimnastas con un IMC similar Body image distortion and eating disorders in adolescent gymnasts vs. a control group of non- gymnasts with similar BMI
}

\author{
*Gabriela Valles Verdugo, *Esteban Hernández Armas, *Raúl Baños, **José Moncada-Jiménez, *Iván Rentería
} Universidad Autónoma de Baja California (México), **Universidad de Costa Rica (Costa Rica)

Resumen. Introducción: Los trastornos de la imagen corporal y alimentarios, así como conductas de control de peso riesgosas para la salud, son más frecuentes en los deportistas que practican deportes con énfasis en la estética, la delgadez y un bajo peso específico. Objetivo: Comparar la imagen corporal (insatisfacción y alteración) y el riesgo de trastornos alimenticios en una muestra de adolescentes gimnastas versus un grupo control de adolescentes no gimnastas con un IMC similar. Material y métodos: Se seleccionó un muestreo no probabilístico por conveniencia de 56 participantes mujeres y hombres clasificados como gimnastas élite, gimnastas regulares y grupo control. La imagen corporal y trastornos alimenticios fueron valorados con la Escala de Factores de Riesgo de Trastornos Alimentarios (EFRATA). Resultados: Al realizar una prueba ANOVA de dos factores, la insatisfacción con la imagen corporal, presentó diferencias estadísticamente significativas por sexo $\left(F_{(1,55)}=17.41, p d » 0.001\right)$ y grupo $\left(F_{(2,55)}=3.66, p=0.033\right)$, mostrando un mayor grado de insatisfacción los hombres $(\mathrm{M}=-0.54)$ respecto a las mujeres $(\mathrm{M}=0.43)$. El riesgo de anorexia mostró diferencias significativas por sexo $\left(\mathrm{F}_{(1,55)}=10.69, \mathrm{p}=0.002\right)$ y grupo $\left(\mathrm{F}_{(2,55)}=7.97, \mathrm{p} \mathrm{d} » 0.001\right)$. Conclusión: Los gimnastas presentaron un mayor grado de insatisfacción corporal al desear una silueta más gruesa o musculosa para el caso de los varones, mientras que las mujeres quieren ser más delgadas respecto al grupo control. De igual forma los gimnastas reportaron un mayor riesgo de anorexia en comparación con el grupo control. Palabras clave: Imagen Corporal, Trastornos Alimentarios, Gimnastas, Índice de Masa Corporal, Insatisfacción Corporal.

\begin{abstract}
Introduction: Body image and eating disorders, as well as risky weight management behaviors are more frequent in athletes who practice sports with an emphasis on aesthetics, thinness and a specific low body weight. Objective: to compare body image (dissatisfaction and alteration) and the risk of eating disorders in a sample of adolescent gymnasts versus a control group of non-gymnastics adolescents with similar BMI. Material and methods: A convenient non-probabilistic sample of 56 female and male participants classified as elite, regular gymnasts and control group with a BMI similar to that of gymnasts. The instrument used to assess body image and eating disorders was the Scale of Risk Factors for Eating Disorders. Results: When performing an inferential analysis using a two-way ANOVA, dissatisfaction with body image showed statistically significant gender $\left(F_{(1,55)}=17.41, p d » 0.001\right)$ and group $\left(F_{(2,55)}=3.66, p=0.033\right)$ differences, males displaying higher degree of dissatisfaction $(\mathrm{M}=-0.54)$ than females $(\mathrm{M}=0.43)$. The risk of anorexia showed significant differences by sex $(F(1,55)=10.69, p=0.002)$ and group $(F(2,55)=7,97, p d » 0.001)$. Conclusion: Gymnasts showed greater body dissatisfaction, with males desiring a more muscular silhouette whilst females a leaner figure compared to the control group. Likewise, gymnasts reported an increased risk of anorexia compared to the control group.
\end{abstract}

Key words: Body Image, Eating Disturbances, Gymnasts, Body Mass Index, Body Dissatisfaction.

\section{Introducción}

La percepción de la imagen corporal en la población adolescente es uno de los temas de mayor auge debido al enaltecimiento de la belleza por parte de los medios de comunicación y la industria dedicada a la apariencia física (Diedrichs, 2012; González, Cuervo, Cachón, \& Zagalaz, 2016; Halliwell, 2013; Levine; 2012; Rosario, 2009; Sypeck, 2006), . El concepto de imagen corporal ha evolucionado a través del tiempo, por lo que ha sido definido de diversas maneras, destacando que es un constructo multidimensional que abarca componentes psicológicos, antropométricos y sociológicos (Gaines \& Burnett, 2014; Meneses-Montero \& MoncadaJiménez, 2008), donde el objeto intencional de reflexión es el propio cuerpo, ya sea a un nivel perceptual, conceptual o emocional, quedando implícita una evaluación emocional sobre el pensamiento y la representación abstracta y parcial del cuerpo humano (Connolly, 2013; Vaquero-Cristóbal, Alacid, Muyor, \& López-Miñarro, 2013). La teoría de la comparación social (Gómez-Peresmitré \& Acosta-García, 2002), señala que la apariencia física puede originar el desarrollo de perturbaciones en la imagen corporal y trastornos

Fecha recepción: 08-08-18. Fecha de aceptación: 17-09-19 Ivan Rentería

irenteria@uabc.edu.mx alimentarios (Bells, Donovan, \& Ramme, 2015), ya que los individuos basan su imagen corporal en comparaciones que realizan de ellos mismos respecto a los demás, viéndose sometidos a una fuerte presión social (Vaquero-Cristóbal et al., 2013), tanto por parte de la familia (Ramos, Pérez, Liberal, \& Latorre, 2003), como de los compañeros de clase (Schaefer \& Salafia, 2014). La conducta alimentaria involucra un conjunto de acciones en respuesta a una estimulación biológica, psicológica y sociocultural vinculada a la ingesta de alimentos; por lo que una alteración de la misma puede originar trastornos alimenticios clínicamente completos o parciales, los cuales, en adolescentes, se desarrollan concomitantemente con la insatisfacción de la imagen corporal (Evans, et al., 2016). En la población adolescente existe un alto riesgo de padecer trastornos de la alimentación, donde se reportan casos de anorexia en edades entre los 13 y 20 años, mientras que la bulimia tiende a desarrollarse en un periodo de edad más tardío (Martínez-Rodríguez et al., 2018; Arévalo et al., 2006); para la distribución de estos padecimientos las mujeres presentan una mayor proporción de trastornos alimenticios respecto a los hombres (Kuczmarski, et al., 2000).

En el ámbito del deporte, donde la delgadez o una silueta musculosa son condiciones de suma importancia para el alto rendimiento, los trastornos alimenticios y de la imagen corporal pueden ser más prevalentes (Kosteli, Van Raalte, Brewer, 
\& Cornelius, 2014; Melin, Torstveit, Burke, Marks, \& Sundgot-Borgen, 2014; Sundgot-Borgen \& Torstveit, 2010). En esta línea, se ha evidenciado que las chicas suelen verse más presionadas hacia una imagen de extrema delgadez (Galli, Reel, Petrie, Greenleaf, \& Carter, 2011), mientas que los chicos suelen sentirse más presionados hacia una imagen musculosa (Tylka, 2011), orientados hacia el aumento de su masa muscular, con ejercicios de fuerza o el uso de complementos alimenticios (McCabe \& Ricciardelli, 2004); sobre todo en chicos que sufren burlas por parte de sus iguales (McVey, Tweed, \& Blackmore, 2005)

Centrándonos en la gimnasia, donde los aspectos estéticos y artísticos pueden influir en el criterio subjetivo de los jueces sobre el desempeño de un atleta, la apariencia física se vuelve un compromiso psicológico para realizar ejercicio ya que el atleta experimenta presión constante por parte de su entrenador, sus compañeros y padres para que mantenga su peso y figura corporal estética con la finalidad de obtener mejores resultados, llevando a los atletas a desarrollar patrones de alimentación poco saludables para satisfacer esas altas demandas de mejores resultados (de Sousa, Mockdece, Fernandes, Sousa, \& Caputo, 2013). Los trastornos de la imagen corporal y alimentarios, así como conductas de control de peso riesgosas para la salud, son más frecuentes en los deportistas que ponen énfasis en la estética, la delgadez y un bajo peso específico que en los deportistas donde estos factores no son tan importantes (Salbach, Klinkowski, Pfeiffer, Lehmkuhl, \& Korte, 2007; Sundgot-Borgen \& Torstveit, 2010).

La falta de información acerca de la prevalencia de insatisfacción de la imagen corporal y su asociación con conductas alimenticias inadecuadas en deportistas de México (Zueck, Muñoz, Chavez, Rodríguez-Villalobos, \& Flores, 2015), se vuelve un factor importante a estudiar por parte de los psicológos del deporte, ya que durante la infancia y adolescencia, el practicar actividades en las que se valoren excesivamente la delgadez y/o el peso se considera como un factor de riesgo para padecer distorsión de la imagen corporal y trastornos alimenticios (Toro, 2004). Por lo anterior, el propósito del presente estudio fue comparar la imagen corporal (insatisfacción y alteración) así como el riesgo de trastornos alimenticios en una muestra de adolescentes gimnastas y un grupo control de adolescentes no gimnastas con un índice de masa corporal (IMC) similar para explorar factores de riesgo asociados a trastornos de carácter psicológico que pudieran presentar atletas que practican el deporte de gimnasia en un alto nivel de rendimiento competitivo. En el presente estudio se planteó como hipótesis que atletas practicantes del deporte de gimnasia a un alto nivel de competencia auto-reportarán una mayor distorsión en la percepción de la imagen corporal y riesgo de trastornos alimenticios respecto a un grupo control de adolescentes no practicantes de gimnasia con un IMC similar.

\section{Material y métodos}

\section{Muestra}

Se realizó un muestreo no probabilístico por conveniencia de 56 sujetos con un rango de edad de entre los 9 a los 18 años, de los cuales 28 eran gimnastas pertenecientes a dos clubes deportivos, donde el Instituto del Deporte y de la Cultura Física del Estado de Baja California, México, selecciona a sus representantes para participar en el proceso de Olimpiada Nacional. Asimismo, se reclutó como grupo control a una muestra de 28 participantes no gimnastas con un IMC similar al de los gimnastas, quienes fueron seleccionados de una institución educativa pública de nivel secundaria en la ciudad de Ensenada, Baja California, México.

Para evaluar a los gimnastas se asistió al gimnasio donde practicaban y de forma individual se le solicitó a cada participante que entregaran un consentimiento informado a sus padres, para que autorizaran su participación en el estudio. Una vez firmado y entregado el documento a los evaluadores se procedió a administrarles el instrumento de evaluación en una sesión de 20 min. En el caso del grupo control, primero se envió una carta dirigida al director de una institución educativa a nivel secundaria con la finalidad de solicitar el acceso a los diferentes sujetos que presentaron un IMC similar al de los gimnastas cuando se evaluó el peso y la estatura de cada uno. Una vez expresada su intención de colaborar en el estudio, se programó, de mutuo acuerdo, una reunión con el personal de la institución y los padres de los sujetos seleccionados para darles a conocer de manera verbal el objetivo y desarrollo del estudio. Posteriormente, se entregó una carta de consentimiento informado a cada padre o madre de los sujetos interesados en participar en el estudio para que proporcionaran su autorización. De esta forma, se siguieron las pautas expresadas en la Declaración de Helsinki para la protección de los derechos de los participantes en una investigación.

\section{Procedimiento}

Antropometría. El peso y la estatura fueron determinados siguiendo los lineamientos de la Sociedad Internacional para el Desarrollo de la Antropometría (ISAK por sus siglas en inglés) especificados en los Estándares Internacionales para la Valoración Antropométrica (Marfell-Jones, Stewart, \& de Ridder, 2012). El peso corporal se midió con una báscula digital marca OMRON Full Body Sensor, modelo HBF514C. Después de haber cotejado que la balanza estuviera calibrada y la pantalla del aparato marcara en cero, se colocó a cada niño en el centro de la báscula, con los brazos a los lados del cuerpo, sin moverse y respirando normalmente. Se registró la medida en kilogramos y un decimal. La estatura máxima se determinó por el método de tracción, en donde el instrumento utilizando fue un estadiómetro portátil marca Seca, modelo 213. El IMC fue calculado por la fórmula de Quetelet $\left(\mathrm{kg} / \mathrm{m}^{2}\right)$ y categorizado de acuerdo a edad y sexo (Kuczmarski, et al., 2000).

Imagen Corporal y Riesgo de Trastornos Alimentarios. El instrumento de evaluación utilizado fue la EFRATA(Escala de Factores de Riesgo Asociados con Trastornos Alimentarios (Gómez-Peresmitré \& Acosta-García, 2000; Gómez-Peresmitré \& Ávila, 1999) y se administró individualmente a cada sujeto en una sesión de 20 min en un aula privada de los gimnasios e institución educativa. El cuestionario contiene diferentes dimensiones que valoran la autoestima e imagen corporal con base en la alimentación, de la siguiente forma. La percepción de la imagen corporal, fue evaluada por un ítem que estima la forma de pensar en el 
adolescente teniendo como opción de respuestas: muy gordo/ gordo/ ni gordo ni delgado/ delgado/ muy delgado. También se evalúo el nivel de insatisfacción o satisfacción con la imagen corporal que se midió a partir de la diferencia entre el valor otorgado a la figura percibida menos el valor de la figura ideal o deseada. Una diferencia igual a cero indica satisfacción, una diferencia positiva representa insatisfacción porque se desea ser más delgado(a), y la diferencia negativa indica también insatisfacción y el deseo de ser más grueso. A su vez, la figura percibida e ideal se miden con un grupo de siluetas que cubren un continuo de peso, desde una figura desnutrida hasta una con obesidad, pasando por una silueta de peso normal. Finalmente, el riesgo de trastorno alimentario fue valorado por medio de una escala tipo Likert con 5 opciones de respuesta, en donde 1 = nunca y 5 = siempre. Esta escala contenía 32 ítems, en los cuales se registra la conducta alimentaria compulsiva, preocupación por el peso y la comida, conducta alimentaria normal, atribución de control alimentario externo, dieta crónica y restringida, conducta alimentaria por compensación psicológica y atribución de control alimentario interno. El riesgo de trastorno alimentario se obtiene del promedio de respuestas, donde los reactivos y puntuaciones altas indicaron un mayor riesgo de sufrir de algún tipo de trastorno alimenticio. Si el promedio es mayor a 1.75 se establece que hay riesgo de desarrollar un trastorno alimentario. Este punto de corte, llamado índice de cambio confiable (ICC), el cual fue propuesto por Jacobson, Follete, y Revenstorf (1984) donde se establece un punto para la evaluación de la significancia clínica (de un nivel de riesgo de trastorno alimentario a uno sin riesgo).

\section{Resultados}

En el estudio participaron 56 adolescentes. El grupo de gimnastas estuvo conformado por 14 mujeres (edad $=10.8 \pm$ 2.1 años, peso $=36.0 \pm 8.5 \mathrm{~kg}$, estatura $=1.39 \pm 0.09 \mathrm{~m}$, IMC $=$ $18.3 \pm 2.1 \mathrm{~kg} / \mathrm{m}^{2}$ ) y 14 hombres (edad $=14.1 \pm 2.2$ años, peso $=49.3 \pm 12.7 \mathrm{~kg}$, estatura $=1.56 \pm 0.12 \mathrm{~m}, \mathrm{IMC}=19.7 \pm 2.2 \mathrm{~kg} /$ $\mathrm{m}^{2}$ ). Los integrantes del grupo control de adolescentes no gimnastas con un IMC similar al de los gimnastas fueron 14 mujeres (edad $=13.3 \pm 1.1$ años, peso $=49.3 \pm 9.5 \mathrm{~kg}$, estatura $\left.=1.57 \pm 0.09 \mathrm{~m}, \mathrm{IMC}=19.6 \pm 2.1 \mathrm{~kg} / \mathrm{m}^{2}\right), \mathrm{y} 14$ hombres $(13.2 \pm$ 1.9 años, peso $=47.2 \pm 13.5 \mathrm{~kg}$, estatura $=1.55 \pm 0.17 \mathrm{~m}$, IMC $\left.=19.1 \pm 2.1 \mathrm{~kg} / \mathrm{m}^{2}\right)$.

En la Tabla 1 se pueden observar los porcentajes obtenidos de la muestra estudiada para la variable satisfacción/ insatisfacción corporal. Quienes presentaron una mayor satisfacción con su silueta corporal fueron las mujeres gimnastas élite (57\%) y los hombres gimnastas no élite (50\%). En el análisis general por sexo, las mujeres desearon ser más delgadas, incluso hasta dos siluetas más en comparación con los hombres, cuya insatisfacción es porque desean estar hasta dos siluetas más gruesos. En independencia del sexo, los más insatisfechos con su figura son los jóvenes no gimnastas. Sin embargo, llama la atención, los altos porcentajes de insatisfacción independientemente de si son o no deportistas de alto rendimiento. Asimismo, es importante resaltar que una cuarta parte de los hombres no gimnastas desearon una figura más delgada, mientras que ninguno de los participantes gimnastas prefirió esta silueta; todos los que mostraron estar insatisfechos desearon ser más gruesos o musculosos.

\section{Análisis Estadístico}

Todos los datos fueron capturados en el programa SPSS, versión 20 para Windows. Se obtuvieron las frecuencias para todas las variables y sus valores porcentuales. Para el análisis estadístico, los grupos fueron clasificados en tres categorías: a) los gimnastas que obtuvieron una medalla en la olimpiada nacional 2014 fueron clasificados como grupo de gimnastas de élite, b) gimnastas regulares, y c) grupo control de adolescentes no gimnastas. Las diferencias entre grupos fueron evaluadas por medio de análisis de varianza (ANOVA), donde se consideró como variables dependientes la satisfacción/ insatisfacción con la imagen corporal y el riesgo de trastorno de la conducta alimentaria, y como variables independientes los grupos de práctica del deporte y el sexo. Se obtuvieron correlaciones producto-momento de Pearson para examinar las relaciones entre la insatisfacción corporal y la composición corporal. El nivel de significancia fue establecido a priori en $\mathrm{p} d » 0.05$.
Tabla 1.

Distribución porcentual de la variable satisfacción, según la relación del grupo de adolescentes y sexo. Los valores de las celdas representan \%. representan \%.

\begin{tabular}{|c|c|c|c|c|c|c|c|c|}
\hline \multirow{2}{*}{ Satisfacción } & \multicolumn{2}{|c|}{ No gimnasta } & \multicolumn{2}{|c|}{ Gimnasta élite } & \multicolumn{2}{|c|}{ Gimnasta } & \multicolumn{2}{|c|}{ Todos } \\
\hline & Femenino & Masculino & Femenino & Masculino & Femenino & Masculino & Femenino & Masculino \\
\hline Más gruesa(o) 2 siluetas & & 8.3 & & 14.3 & & & & 10.7 \\
\hline Más gruesa(o) 1 silueta & 14.3 & 33.3 & 28.6 & 50.0 & & 50.0 & 14.3 & 42.9 \\
\hline Satisfecha(o) & 42.9 & 33.3 & 57.1 & 35.7 & 42.9 & 50.0 & 46.4 & 35.7 \\
\hline Más delgada(o) 1 silueta & 28.6 & 25.0 & 14.3 & & 14.3 & & 21.4 & 10.7 \\
\hline Más delgada(o) 2 siluetas & 14.3 & & & & 42.9 & & 17.9 & \\
\hline
\end{tabular}

Tabla 2.

Distribución porcentual de la variable alteración de la imagen corporal, según la relación con el grupo de adolescentes y sexo. Los valores de las celdas representan $\%$.

\begin{tabular}{|c|c|c|c|c|c|c|c|c|}
\hline \multirow[t]{2}{*}{ Grado de alteración } & \multicolumn{2}{|c|}{ No gimnasta } & \multicolumn{2}{|c|}{ Gimnasta élite } & \multicolumn{2}{|c|}{ Gimnasta } & \multicolumn{2}{|c|}{ Todos } \\
\hline & Femenino & Masculino & Femenino & Masculino & Femenino & Masculino & Femenino & Masculino \\
\hline Subestimación grado 2 & & 8.3 & & & & & & 3.6 \\
\hline Subestimación grado 1 & 42.9 & 41.7 & 14.3 & 78.6 & 14.3 & & 28.6 & 57.1 \\
\hline No alterado & 57.1 & 41.7 & 85.7 & 14.3 & 71.4 & 100.0 & 71.4 & 32.1 \\
\hline Sobreestimación grado 1 & & 8.3 & & 7.1 & & & & 7.1 \\
\hline
\end{tabular}

Tabla 3.

Efectos del grupo de adolescentes y el sexo en la satisfacción/insatisfacción de la silueta corporal de acuerdo al sexo y por grupo estudiado.

\begin{tabular}{|c|c|c|c|c|c|}
\hline Origen & Suma de cuadrados tipo I & $\mathrm{gl}$ & Media cuadrática & $\mathrm{F}$ & Sig. \\
\hline Modelo corregido & $19.446^{\mathrm{a}}$ & 5 & 3.889 & 5.201 & .001 \\
\hline Intersección & .161 & 1 & .161 & .215 & .645 \\
\hline Sexo & 13.018 & 1 & 13.018 & 17.407 & .000 \\
\hline Grupo de Adolescentes & 5.470 & 2 & 2.735 & 3.657 & .033 \\
\hline Sexo * Grupo de Adolescentes & .958 & 2 & .479 & .641 & .531 \\
\hline Error & 37.393 & 50 & .748 & & \\
\hline Total & 57.000 & 56 & & & \\
\hline Total corregida & 56.839 & 55 & & & \\
\hline \multicolumn{6}{|c|}{ a. $\mathrm{R}$ cuadrado $=.342(\mathrm{R}$ cuadrado corregida $=.276)$} \\
\hline \multicolumn{6}{|c|}{$\begin{array}{l}\text { Tabla } 4 . \\
\text { Efectos del grupo de adolescentes y el sexo en la ingesta alimentaria por compensación psicológica de acuerdo al sexo y por grupo } \\
\text { estudiado. }\end{array}$} \\
\hline Origen & Suma de cuadrados tipo I & gl & Media cuadrática & $\mathrm{F}$ & Sig. \\
\hline Modelo corregido & $.946^{\mathrm{a}}$ & $\frac{81}{5}$ & 189 & .638 & .672 \\
\hline Intersección & 128.503 & 1 & 128.503 & 433.460 & .000 \\
\hline Sexo & .043 & 1 & .043 & .144 & .706 \\
\hline Grupo de Adolescentes & .017 & 2 & .008 & .028 & .973 \\
\hline Sexo* Grupo de Adolescentes & .886 & 2 & .443 & 1.495 & .234 \\
\hline Error & 14.823 & 50 & .296 & & \\
\hline Total & 144.272 & 56 & & & \\
\hline Total corregida & 15.769 & 55 & & & \\
\hline
\end{tabular}


En cuanto a la alteración de la imagen corporal, en la Tabla 2 se observa que las mujeres tienen una menor alteración de su imagen corporal en comparación con los hombres, quienes muestran una mayor subestimación y un bajo porcentaje de sobrestimación. El 8\% de los no gimnastas y el $7 \%$ de los gimnastas élite se perciben más gruesos de lo que en realidad son. Por otra parte, en el caso de los gimnastas élite, existe una diferencia considerable, ya que las mujeres se perciben en un $86 \%$ como en realidad son y un $79 \%$ de los hombres se perciben más delgados de cómo en realidad se encuentran.

La prueba deANOVA indica que no existe una interacción significativa entre los grupos y el sexo en la satisfacción/ insatisfacción de la silueta corporal ( $p=.531$ ). Se encontraron efectos principales significativos, que indican diferencias en la satisfacción/insatisfacción de la silueta corporal en función del grupo de deportistas ( $p=.033$ ) y el sexo de los participantes ( $p \mathrm{~d} » .001)$.

De igual forma, no existe una interacción significativa entre los grupos y el sexo en la en la ingesta alimentaria por compensación psicológica $(p=.234)$. Tampoco se encontraron efectos principales significativos que indicaran diferencias en la ingesta alimentaria por compensación psicológica en función del grupo de deportistas ( $p=.973$ ) y el sexo de los participantes $(p=.706)$.

\section{Discusión}

El propósito del estudio fue comparar la imagen corporal y el riesgo de trastornos alimenticios en adolescentes gimnastas y controles de Baja California, México. La comparación de factores de riesgo asociados a la imagen corporal en deportistas que se dedican a deportes estéticos respecto a un grupo control de no deportistas, adquiere importancia desde un punto de vista teórico y práctico. La evidencia científica sugiere que este tipo de deportistas se enfrenta a niveles de presión constante por parte de sus entrenadores y familiares para mantener una silueta delgada y estética, y este tipo de coacciones los vuelve más propensos a desarrollar alteraciones de la imagen corporal y trastornos alimenticios (Krane, Waldron, Michalenok, \& Stiles-Shipley, 2001; Petrie \& Stoever, 1993; Salbach, et al., 2007).

Los principales hallazgos de este estudio apoyan la noción de que en comparación con sujetos controles no deportistas, el grupo de gimnastas presentó una mayor frecuencia en alteraciones de la imagen corporal, ya que las mujeres querían ser más delgadas y los hombres más musculosos; sin embargo, no se encontró algún otro efecto significativo respecto a la insatisfacción de la imagen corporal. Este hallazgo es similar al encontrado en bailarinas de ballet y fisicoculturistas al compararlos contra un grupo control de usuarios de gimnasios (Ravaldi, et al., 2003). Esto puede demostrar que la insatisfacción corporal en los sujetos evaluados se relaciona con el deporte practicado, de hecho, el grado de delgadez que señalan las mujeres gimnastas está correlacionado con el nivel de desempeño, ya que las gimnastas élite fueron las que reportaron una mayor tendencia a querer tener una silueta corporal que luciera con mayor delgadez. Esto posiblemente pueda deberse a un estereotipo de masculinización por efecto del alto grado de realiza- ción de actividad física (Hardin \& Greer, 2009). Los hombres reportaron una silueta menos musculosa, por lo que catalogaron su físico como el no ideal. Las preocupaciones por la imagen corporal, tradicionalmente se han visto como una preocupación que afecta en un mayor grado a la mujer (Galli et al., 2011); no obstante, los resultados de este estudio señalan que ambos grupos de atletas se preocupan por igual en términos de la imagen corporal, ya que los hombres también son vulnerables a presiones sociales y culturales en cuanto a mantener una silueta corporal estética, difiriendo de la mujer en términos de querer tener un físico más musculoso y masculino, pudiendo deberse a un estereotipo de masculinización por efecto del alto grado de realización de actividad física (Leit, Gray, \& Pope, 2002).

En cuanto a los hábitos alimenticios, las gimnastas élite femeniles se detectó una diferencia significativa respecto al grupo control en la variable de dieta crónica restrictiva, lo que podría influir a futuro en su rendimiento físico debido a realizan actividad física intensa por más de 10 a 20 h a la semana. Sin embargo, en un meta análisis reportado por Hausenblas y Downs (2001), no se encontraron diferencias significativas en la imagen corporal y trastornos alimenticios al comparar gimnastas de nivel élite respecto a un grupo control de no deportistas, concluyendo que dichos factores de riesgo no presentaron diferencias significativas debido a que posiblemente los gimnastas poseen una mejor salud mental que los no deportistas, por lo que posiblemente tienen una mayor capacidad para hacer frente a las presiones de mantener un bajo peso, así como una silueta estética y al mismo tiempo someterse a grandes cargas de actividad física.

El estudio presenta factores limitantes importantes. El primero, es que el muestreo no es representativo de los gimnastas y adolescentes del estado de Baja California. Segundo, el diseño del estudio es transversal, lo que dificulta formular declaraciones concluyentes sobre la causalidad de los resultados. Tercero, el IMC es una herramienta subjetiva para valorar el estado nutricional de un sujeto, pues se prefiere un método más exacto como por ejemplo, la absorciometría de energía dual de rayos X (DXA) (Nana, Slater, Stewart, \& Burke, 2015). De igual forma, la edad se considera una limitante en el estudio debido a que la mayoría de los sujetos evaluados se encontraba entre la tercera etapa de niñez y la etapa de pre-adolescencia, donde ocurren un sinnúmero de cambios en la composición corporal por efecto de la maduración y crecimiento, no obstante, se ha reportado en la literatura científica (Neves et al., 2017) que la principal insatisfacción con la imagen corporal que presentan los sujetos a esa edad es la tendencia lipofóbica, siendo este comportamiento consistente hasta la adultez media. Por otra parte, al valorar la imagen corporal en niños de la tercera otro factor importante que se presenta de manera uniforme al evaluar la insatisfacción corporal es el aspecto cultural, donde la tendencia cultural a considerar la delgadez como el patrón corporal ideal para las mujeres (Martínez-Rodríguez et al., 2018) y para los hombres, la musculatura es el patrón deseado (Vaquero-Cristobal et al., 2013). La evaluación negativa de la imagen corporal en la mayoría de los casos se produce debido a la dificultad de montaje en estos modelos (Neves et al., 2017) y no por efecto de la edad cronológica, por lo que 
en el presente estudio se obtuvieron resultados similares a lo reportado por Neves et al. (2017) en su revisión literaria.

Por otra parte, una fortaleza del presente estudio fue que los sujetos al momento de auto administrarles el cuestionario, las condiciones ofrecidas por los responsables del estudio fueron de privacidad, anonimato y confidencialidad, lo que pudo haber ayudado a los sujetos para que no subestimaran o sobreestimaran el nivel de sus respuestas, además de que el cuestionario utilizado ha mostrado una alta validez y confiabilidad para evaluar los constructos estudiados (Gómez-Peremistré \& Acosta-García, 2002).

Con base en los resultados obtenidos se sugiere implementar programas de promoción de la salud enfocados en atención psicológica sobre aspectos negativos de la imagen corporal y trastornos alimenticios dirigidos a hombres y mujeres adolescentes. Además, debería prestarse una mayor atención al estudio de la imagen corporal en adolescentes varones respecto a un grupo control, ya que en este estudio presentaron al igual que las mujeres, una insatisfacción de la imagen corporal; sin embargo, un amplio cuerpo de la literatura moderna ha prestado una mayor atención al estudio de la imagen corporal y trastornos alimenticios en las mujeres que se dedican a deportes o actividades estéticas.

\section{Conclusión}

Los atletas no están sometidos a una presión psicológica diferente que la de sujetos normales de su misma edad; sin embargo, aunque las mujeres manifestaron sentirse conformes con su imagen corporal actual, presentaron un bajo factor de riesgo para sufrir algún trastorno del comportamiento alimentario, lo que pudiera ser un indicador de que el género parece moderar la relación de la imagen corporal y trastornos alimenticios entre los jóvenes. Por otra parte, aunque la mayoría de los atletas evaluados registraron un «rango saludable» en las variables estudiadas, los presentes resultados sugieren que la preocupación sobre su imagen corporal y dietas crónicas restringidas, sobre todo en los gimnastas élite puede estar justificada debido a la presión constante que sufren por parte de sus entrenadores y familiares para mantenerse en dicho estatus. Para finalizar, los resultados de este estudio deben ser confirmados por estudio que contenga un diseño longitudinal y un tamaño de muestra que sea representativo de los adolescentes.

\section{Referencias}

Arévalo, R. V., Aguilar, X. L., Rayón, G. L. Á., Díaz, J. M. M. y Ruiz, A. O. (2006). Insatisfacción corporal e influencia de los modelos estéticos en niños y jóvenes varones mexicanos. Enseñanza e investigación en Psicología, 11(1), 185-197.

Bell, H. S., Donovan, C. L., \& Ramme, R. (2016). Is athletic really ideal? An examination of the mediating role of body dissatisfaction in predicting disordered eating and compulsive exercise. Eating behaviors, 21, 24-29. doi: http://doi.org/cstt

Connolly, A. (2013). Out of the body: embodiment and its vicissitudes. Journal of Analytical Psychology, 58(5), 636-656. doi: http://doi.org/f5hrnf
De Souza-Fortes, L. D. S., Neves, C. M., Filgueiras, J. F., Almeida, S. S., \& Ferreira, M. E. C. (2013). Body dissatisfaction, psychological commitment to exercise and eating behavior in young athletes from aesthetic sports. Revista Brasileira de Cineantropometria \& Desempenho Humano, 15(6), 695-704. doi: http://doi.org/ cstr

Diedrichs, P. C. (2012). Media influences on male body image. In T. Cash (Eds) Encyclopedia of body image and human appearance. Oxford: Academic.

Evans, E. H., Adamson, A. J., Basterfield, L., Le Couteur, A., Reilly, J. K., Reilly, J. J., \& Parkinson, K. N. (2017). Risk factors for eating disorder symptoms at 12 years of age: A 6-year longitudinal cohort study. Appetite, 108, 12-20. doi: http://doi.org/csts

Gaines, S. A., \& Burnett, T. B. S. (2014). Perceptions of eating behaviors, body image, and social pressures in female division II college athletes and non-athletes. Journal of Sport Behavior, 37(4), 351-369.

Galli, N., Reel, J. J., Petrie, T., Greenleaf, C., \& Carter, J. (2011). Preliminary development of the weight pressures in sport scale for male athletes. Journal of Sport Behavior, 34(1), 47-68.

Gómez-Peresmitré, G y Acosta-García, M. V. (2000). Imagen corporal como factor de riesgo en los trastornos de la alimentación: una comparación transcultural entre México y España. Clínica y salud, 11(1).

Gómez-Peresmitré, G., \& Acosta-García, M. V. (2002). Valoración de la delgadez. Un estudio transcultural (México/ España). Psicothema, 14(2), 221-226.

Gómez-Peresmitré, G. y Ávila, E. (1999). Preadolescentes mexicanas y cultura de la delgadez: Figura ideal anoréctica y preocupación excesiva por el peso corporal. Revista Mexicana de Psicología, 1(6), 153-166.

González, C., Cuervo, C., Cachón, J. y Zagalaz, M. L. (2016). The relationship between demographic variables, physical exercise practice and perception of the body image of undergraduate student teachers. Retos, Nuevas Tendencias en Educación Física Deporte y Recreacion, 29, 90-94.

Halliwell, E. (2013). The impact of thin idealized media images on body satisfaction: Does body appreciation protect women from negative effects?. Body Image, 10(4), 509514. doi: http://doi.org/f5fsg7

Hardin, M., \& Greer, J. D. (2009). The Influence of Genderrole Socialization, Media Use and Sports Participation on Perceptions of Gender-Appropriate Sports. Journal of Sport Behavior, 32(2) 207-228.

Hausenblas, H. A., \& Downs, D. S. (2001). Comparison of body image between athletes and nonathletes: A metaanalytic review. Journal of Applied Sport Psychology, 13(3), 323-339. doi: http://doi.org/fbm7s8

Kosteli, M. C., Van Raalte, J. L., Brewer, B. W., \& Cornelius, A. E. (2014). Relationship between sport type and body image of female athletes. TRENDS in Sport Sciences, 21(2), 65-72. Recuperado de: http:// www.tss.awf.poznan.pl/files/TRENDS_1_2014_2.pdf

Krane, V., Waldron, J., Michalenok, J., \& Stiles-Shipley, J. (2001). Body image concerns in female exercisers and athletes: A feminist cultural studies perspective. Women 
in Sport and Physical Activity Journal, 10(1), 17-54. doi: http://doi.org/cstk

Kuczmarski, R.J., Ogden, C.L., Grummer-Strawn, L.M., Flegal, K.M., Guo, S.S., Wei, R., ... Johnson, M. (2000). CDC growth charts: United States. Advance data 2000 (314):127. Recuperado de: https://www.cdc.gov/nchs/data/ad/ ad314.pdf

Leit, R. A., Gray, J. J., \& Pope Jr, H. G. (2002). The media’s representation of the ideal male body: A cause for muscle dysmorphia?. International Journal of Eating Disorders, 31(3), 334-338. doi: http://doi.org/fdbz4m

Levine, M. P. (2012). Media influences on male body image. In T. Cash (Eds) Encyclopedia of body image and human appearance. Oxford: Academic

Marfell-Jones, M. J., Stewart, A. D., \& De Ridder, J. H. (2012). International standards for anthropometric assessment. Wellington, New Zealand: International Society for the Advancement of Kinanthropometry. Recuperado de: http://hdl.handle.net/11072/1510

McCabe, M., \& Ricciardelli, L. (2004). A longitudinal study of pubertal timing and extreme body change behaviors among adolescent boys and girls. Adolescence, 39(153), 145-166. https://doi.org/10.1002/erv.389

McVey, G., Tweed, S., \& Blackmore, E. (2005). Correlates of weight loss and muscle-gaining behavior in 10-to 14year-old males and females. Preventive medicine, 40(1), 1-9. https://doi.org/10.1016/j.ypmed.2004.04.043

Melin, A., Torstveit, M. K., Burke, L., Marks, S., \& SundgotBorgen, J. (2014). Disordered eating and eating disorders in aquatic sports. International Journal of Sport Nutrition and Exercise Metabolism, 24(4), 450-459. doi: http://doi.org/f6ftjv

Meneses-Montero, M. y Moncada-Jiménez, J. (2008). Imagen corporal percibida e imagen corporal deseada en estudiantes universitarios. Revista Iberoamericana de Psicología del Ejercicio y el Deporte, 3(1), 13-30.

Nana, A., Slater, G. J., Stewart, A. D., \& Burke, L. M. (2015). Methodology review: using dual-energy X-ray absorptiometry (DXA) for the assessment of body composition in athletes and active people. International Journal of Sport Nutrition and Exercise Metabolism, 25(2), 198-215. doi: http://doi.org/f69ddx

Neves, C. M., Cipriani, F. M., Meireles, J. F. F., da Rocha Morgado, F. F., \& Ferreira, M. E. C. (2017). Body image in childhood: An integrative literature review. Revista Paulista de Pediatria, 35(3), 331.

Nieves, I. R. (2009). La imagen corporal: hacia una construcción social para la Psicología Industrial Organizacional. Poiésis, 9(18). doi: https://doi.org/ 10.21501/16920945.131

Petrie, T. A., \& Stoever, S. (1993). The incidence of bulimia nervosa and pathogenic weight control behaviors in female collegiate gymnasts. Research Quarterly for Exercise and Sport, 64(2), 238-241. doi: http://doi.org/ cstj

Ramos, P., Pérez, L., Liberal, S., \& Latorre, M. (2003). La imagen corporal en relación con los TCA en adolescentes vascos de 12 a 18 años. Revista de Psicodidáctica, 15(16), 65-74.

Ravaldi, C., Vannacci, A., Zucchi, T., Mannucci, E., Cabras, P.
L., Boldrini, M., ... \& Ricca, V. (2003). Eating disorders and body image disturbances among ballet dancers, gymnasium users and body builders. Psychopathology, 36(5), 247-254. doi: http:// doi.org/cc62k3

Salbach, H., Klinkowski, N., Pfeiffer, E., Lehmkuhl, U., \& Korte, A. (2007). Body image and attitudinal aspects of eating disorders in rhythmic gymnasts. Psychopathology, 40(6), 388-393. doi: http:// doi.org/fmx4tr

Schaefer, M. K., \& Salafia, E. H. B. (2014). The connection of teasing by parents, siblings, and peers with girls' body dissatisfaction and boys' drive for muscularity: The role of social comparison as a mediator. Eating behaviors, 15(4), 599-608. https://doi.org/10.1016/ j.eatbeh.2014.08.018

Sundgot Borgen, J., \& Torstveit, M. K. (2010). Aspects of disordered eating continuum in elite high intensity sports. Scandinavian Journal of Medicine \& Science in Sports, 20, 112-121. doi: http://doi.org/fxfsmb

Sypeck, M. F., Gray, J. J., Etu, S. F., Ahrens, A. H., Mosimann, J. E., \& Wiseman, C. V. (2006). Cultural representations of thinness in women, redux: Playboy magazine's depiction of beauty from 1979 to 1999. Body image, 3(3), 229-235. doi: http://doi.org/c8ddth

Tan, J., Bloodworth, A., McNamee, M., \& Hewitt, J. (2014). Investigating eating disorders in elite gymnasts: Conceptual, ethical and methodological issues. European Journal of Sport Science, 14(1), 60-68. doi: http://doi.org/ cstq

Taylor, N. L. (2011). «Guys, she’s humongous!»: Gender and weight-based teasing in adolescence. Journal of Adolescent Research, 26(2), 178-199. https://doi.org/ 10.1177/0743558410371128

Toro, J. (2004). Riesgo y causas de la anorexia nerviosa. Barcelona: Ariel.

Vaquero-Cristóbal, R., Alacid, F., Muyor, J. M. y LópezMiñarro, P. Á. (2013). Body image; literature review. Nutricion Hospitalaria, 28(1), 27-35. doi: http:// doi.org/fbm7s8

Zueck, M.C., Muñoz, F., Chavez, A., Rodriguez-Villalobos, J.M. y Flores, F.J. (2015). Physical Self-Concept Differences in Young Male Mexican Athletes and NonAthletes. American Journal of Applied Psychology; 4(1), 9-12. doi: http://doi.org/cstp

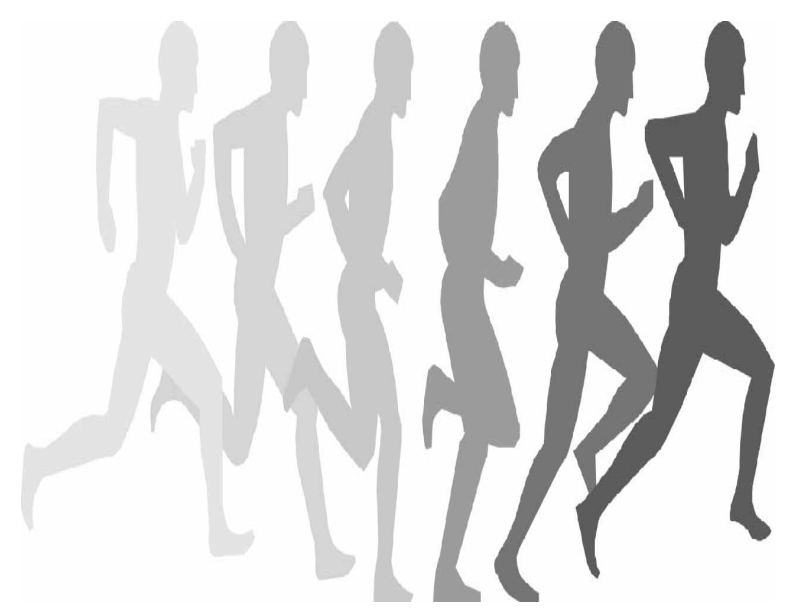

